\title{
X-Ray Scattering Studies of HgSe Nanoclusters in Zeolite
}

\author{
A.M.M. ABEYKOON, M. CASTRO-COLIN, E.V. ANOKHINA, M.N. ILIEV, \\ W. DONNER, M. BRUNELLI, A.J. JACOBSON, and S.C. MOSS
}

\begin{abstract}
Structural studies done on $\mathrm{HgSe}$ nanoclusters fabricated in the pores of two types of zeolite frameworks, LTL (tubular pore) and Nd-Y (nearly spherical pore), are presented. Nanoclusters were synthesized in these zeolite frameworks via chemical vapor deposition. Anomalous X-ray scattering (AXS) studies were performed on both $\mathrm{HgSe} /$ zeolite-Y and $\mathrm{HgSe} /$ zeolite-L systems. As a result, we present two distinct structural models for $\mathrm{NdY} / \mathrm{HgSe}$ and $\mathrm{LTL} / \mathrm{HgSe}$ systems. A remarkable feature present in our X-ray diffraction (XRD) patterns, diffuse scattering, will also be discussed using our initial results of the atomic pair distribution function (PDF) studies. X-ray structural work is complemented using the results of optical and Raman studies.
\end{abstract}

DOI: $10.1007 / \mathrm{s} 11661-008-9563-9$

(C) The Minerals, Metals \& Materials Society and ASM International 2008

\section{INTRODUCTION}

NANOSTRUCTURED semiconductors exhibit novel properties due to the spatial confinement of the charge carriers in discrete energy levels, which is known as the size quantization effect. This provides us an opportunity to observe and control the properties of these materials as they evolve from atoms or molecules to the bulk. The ability to control electronic, magnetic, optical, and photocatalytic properties of these nanostructures makes them ideal candidates to be employed in the electronic and optoelectronic industry. ${ }^{[1,2]}$ Studying the atomic structures of these nanoclusters is necessary for an analysis of the transition from molecular to bulk semiconductor properties.

Fabrication of semiconductor nanoclusters in a wellorganized host matrix is a promising strategy to form semiconductor nanoclusters with a highly uniform shape and size distribution. ${ }^{[3-9]}$ Rigidity of the framework,

A.M.M. ABEYKOON, Postdoctoral Fellow, is with the Department of Physics, University of Houston, Houston, TX 77204. Contact e-mail: aabeykoon@UH.EDU M. CASTRO-COLIN, formerly with the Department of Physics, University of Houston, is Assistant Professor, Department of Physics, University of Texas, El Paso, TX 79968. E.V. ANOKHINA, formerly with the Department of Chemistry, University of Houston, is deceased. M.N. ILIEV, Research Professor, is with the Texas Center for Superconductivity at the University of Houston (TcSUH), Houston, TX 77204. W. DONNER, formerly with the Department of Physics, University of Houston, is Professor, Technishe Universitat Darmstadt Fachbereich Materialund Geowissenschaften-Srukturforschung Petersenstrasse 23, 64287 Darmstadt, Germany. M. BRUNELLI, Scientist, is with the European Synchrotron Radiation Facility (ESRF), 38043 Grenoble, France. A.J. JACOBSON, Robert A. Welch Professor of Chemistry and the Director of the Texas Center for Superconductivity, at the University of Houston (TcSUH). S.C. MOSS, Professor (Emeritus), is with the Department of Physics, University of Houston.

This article is based on a presentation given in the symposium entitled "Neutron and X-Ray Studies for Probing Materials Behavior," which occurred during the TMS Spring Meeting in New Orleans, LA, March 9-13, 2008, under the auspices of the National Science Foundation, TMS, the TMS Structural Materials Division, and the TMS Advanced Characterization, Testing, and Simulation Committee. Article published online June 12, 2008 uniform pore size, and their ability to adsorb molecular species make zeolites ideal candidates to accommodate organic and inorganic molecular species. Being transparent in a wide spectral range is another advantage associated with zeolites.

Conventional X-ray and neutron diffraction, ${ }^{[10]}$ anomalous X-ray scattering (AXS), ${ }^{[6,11]}$ the pair distribution function (PDF) technique using synchrotron $\mathrm{X}$-rays or neutrons, ${ }^{[12]}$ and Raman spectroscopy ${ }^{[4,10,13]}$ are some of the techniques capable of extracting structural information from the encapsulated nanoclusters in zeolites. The AXS is a very powerful technique to obtain structural information beyond the nearest neighbor, especially when one phase is embedded in another ${ }^{[6,11]}$ In this method, multiple X-ray diffraction (XRD) patterns are recorded, one at the vicinity $(-20 \mathrm{eV})$ of the absorption edge of an element of interest and the other one far $( \pm 200 \mathrm{eV})$ from the absorption edges. Then, the structure factor associated with the particular element can be determined by exploiting the difference in scattering power of the element at the two energies. As a result, the identity of the element at each site can ideally be attained. In addition, if there exists disorder within the encapsulate such as orientational, displacive, or substitutional disorder, it will alter the intensities of the Bragg peaks and give rise to diffuse scattering ${ }^{[14]}$ between the Bragg peaks of the XRD pattern. In such cases, the PDF technique is capable of extracting additional structural information from the encapsulate. The PDF is obtained by Fourier transforming the properly corrected and normalized X-ray or neutron data into real space coordinates. ${ }^{[14-17]}$ Because the Fourier transform of data includes both Bragg and diffuse scattered intensity, the PDF produces long-range and short-range order structural information simultaneously, albeit in a one-dimensional (1-D) projection. Once the data are Fourier transformed, a structural model can be refined by fitting the PDF.

At the initial phase of this research, $\mathrm{HgSe}$ nanoclusters were synthesized in two zeolite frameworks, zeolite L (LTL structure so-called Linde type L, which contains 
$7.1 \AA$ diameter tubular pores) and zeolite Y (Faujasite structure, which contains $13 \AA$ diameter nearly spherical pores). At the second phase, the atomic structures of the synthesized nanoclusters were modeled by performing the Rietveld refinement on X-ray data. These results were complemented by optical studies. Our intention here is to summarize our previous work on synthesis and structural studies of $\mathrm{HgSe}$ nanoclusters in zeolite ${ }^{[18]}$ and to show some of our new results on the disordered part of these structures.

\section{NANOCLUSTER SYNTHESIS AND IN-HOUSE CHARACTERIZATION}

Hydrated forms of zeolites, LTL $\left(\mathrm{K}_{9}\left(\mathrm{H}_{2} \mathrm{O}\right)_{x} \mathrm{Si}_{27}\right.$ $\left.\mathrm{Al}_{9} \mathrm{O}_{72}\right)$ and $\mathrm{Na}-\mathrm{Y}\left(\mathrm{Na}_{56}\left(\mathrm{H}_{2} \mathrm{O}\right)_{x} \mathrm{Si}_{136} \mathrm{Al}_{56} \mathrm{O}_{384}\right)$ (from Zeolyst), were used for our study. A Nd ionic exchange was performed on $\mathrm{Na}-\mathrm{Y}$ to remove $\mathrm{Na}$ cations from the main cavities. Nanoclusters were synthesized in dehydrated zeolite cavities, tubular channels of LTL, and nearly spherical cavities of NdY, via chemical vapor deposition. Details of the synthesis procedure are described elsewhere. ${ }^{[18]}$

Band gaps of the incorporated nanoclusters were estimated using optical absorption spectra. We observed a clear increase in "band gap" with decreasing particle size obeying the size quantization effect. ${ }^{[19]}$ The estimated band gaps for $\mathrm{HgSe}$ clusters in zeolites LTL (contains $7.1 \AA$ diameter 1-D channels) and $\mathrm{Nd}-\mathrm{Y}$ (contains $13 \AA$ diameter nearly spherical cages) were 2.25 and $2.03 \mathrm{eV}$, respectively. In describing the electronic structure of these nanoclusters, the usual $E(\bar{k})$, the wave vector $\bar{k}$-energy dispersion, is not meaningful because $\bar{k}$ is not a proper quantum number. As the molecules increase in size toward the bulk, a proper $\bar{k}$ dependent band gap may be used. The transition from "HOMO-LUMO" notation to band gap, however, is apparently smooth.

The Raman spectrum of LTL/HgSe gave three clearly pronounced peaks at 180, 202, and $263 \mathrm{~cm}^{-1}$ (Figure 1). For comparison, the Raman spectrum of Se-incorporated LTL, exhibiting a single peak at $263 \mathrm{~cm}^{-1}$, is also shown in the figure. A similar Raman peak at $263 \mathrm{~cm}^{-1}$

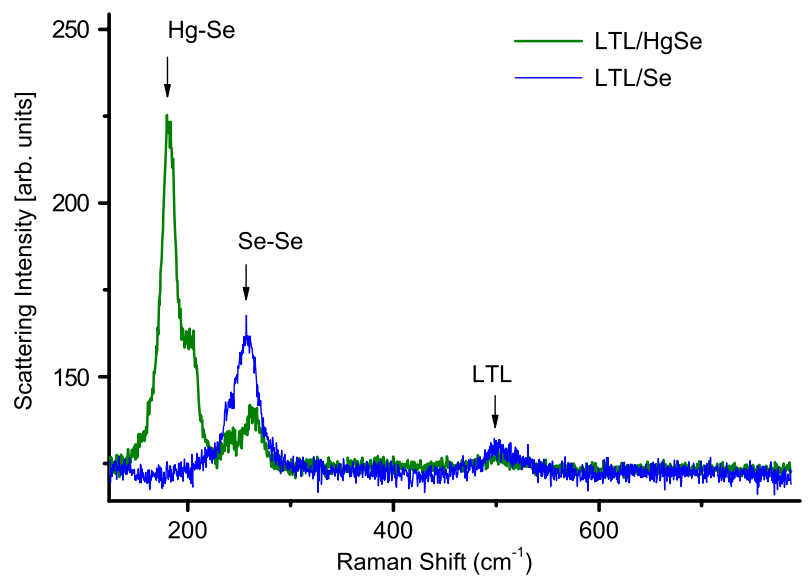

Fig. 1-Raman spectra of LTL/Se and LTL/HgSe systems.

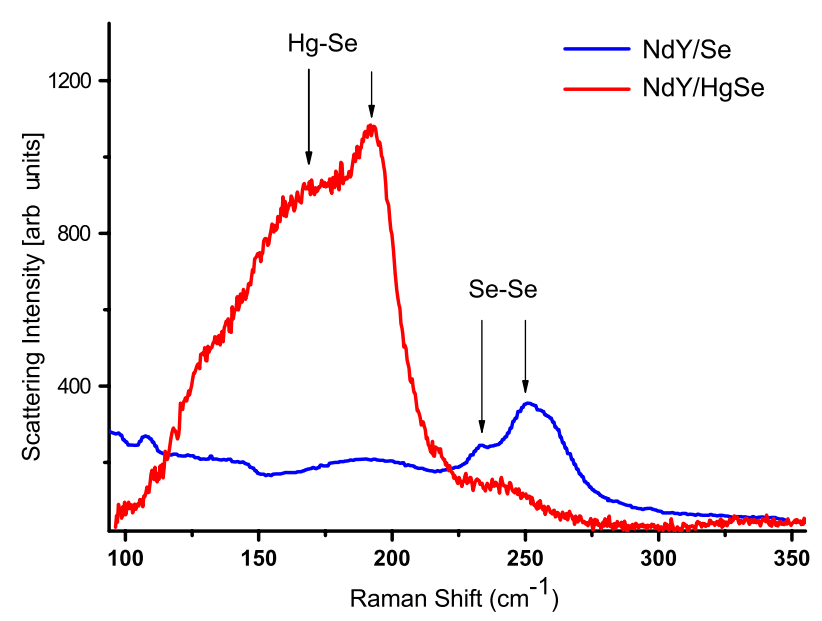

Fig. 2-Raman spectra of $\mathrm{NdY} / \mathrm{Se}$ and $\mathrm{NdY} / \mathrm{HgSe}$ systems.

was reported earlier for Se incorporated $\mathrm{AlPO}_{4}-5$ single crystals. ${ }^{[10,13]}$ Because the dimensions of LTL and $\mathrm{AlPO}_{4}-5$ channels are very close, the $263 \mathrm{~cm}^{-1}$ peak can unambiguously be assigned to $\mathrm{Se}$ in the LTL channels. The Raman features at 180 and $202 \mathrm{~cm}^{-1}$, which are absent for empty zeolite and LTL/Se, cannot be associated with either bulk $\mathrm{Se}$ or $\mathrm{HgSe}$ (they do not correspond to the bulk HgSe Raman peaks). This suggests that they originate from the unique $\mathrm{HgSe}$ nanostructure, which differs in the two zeolites studied.

Figure 2 shows the Raman spectra of $\mathrm{Nd}-\mathrm{Y} / \mathrm{HgSe}$ and $\mathrm{Nd}-\mathrm{Y} / \mathrm{Se}$ systems. As with the LTL/HgSe, the peaks at 168 and $192 \mathrm{~cm}^{-1}$ for $\mathrm{Nd}-\mathrm{Y} / \mathrm{HgSe}$ can be associated with the formation of $\mathrm{Hg}$-Se nanoclusters in the pores of $\mathrm{Nd}-\mathrm{Y}$, whereas the peak near $238 \mathrm{~cm}^{-1}$ can be assigned to disordered Se chains ${ }^{6}$ or polyselenide anions such as $\mathrm{Se}_{2}^{-2} \cdot[20]$

\section{XRD AND THE RIETVELD REFINEMENT}

Zeolites, LTL, and Nd-Y have well-organized structures resulting in well-resolved and sharp Bragg reflections. ${ }^{[21-25]}$ Incorporation of nanoclusters, however, changes Bragg intensities of the zeolite XRDs without giving rise to new peaks. This indicates that the zeolite framework symmetries remain intact with the incorporation of $\mathrm{Hg}$ and $\mathrm{Se}$ atoms, and these atoms occupy well-defined positions in the framework. It should be noted that if the nanocluster symmetry is everywhere different from the host, extra intensities will appear. However, incorporation of nanoclusters introduces significant diffuse scattering under the Bragg peaks, indicating disorder within the individual clusters representing deviations from the average structure obtained by the Rietveld analysis. This diffuse scattering background was subtracted for the Rietveld analysis.

A structural model was refined simultaneously against anomalous X-ray data sets collected at $\mathrm{Hg}$-L edge$20 \mathrm{eV}$, Se-K edge- $20 \mathrm{eV}$, and off-edge energies introducing corresponding $f^{\prime}$ and $f^{\prime \prime}$ values ${ }^{[18,26]}$ for the real and imaginary contributions to the dispersion correc- 
tion. Anomalous contrast at the near edge data sets of the atomic structure factor was used to properly locate $\mathrm{Hg}$ and $\mathrm{Se}$ atoms in zeolite cavities. Details of the refinement procedure and the resulting atomic parameters are given in Abeykoon et al. ${ }^{[18]}$ Schematic diagrams of the refined structural models for the nanoclusters in LTL channels and NdY cages are presented in Figures 3 and 4, respectively. The Hg1Se $3=2.707 \AA$ distance is reasonable and close to the bulk $\mathrm{Hg}$-Se distance, $\sim 2.64 \AA$ in the refined nanostructure inside LTL channels. For the refined $\mathrm{HgSe}$ nanocluster in NdY cages, Se-Hg $(2.67 \AA)$ and Se-Se $(2.31 \AA)$ distances are reasonable and close to the bulk $\mathrm{Hg}$-Se

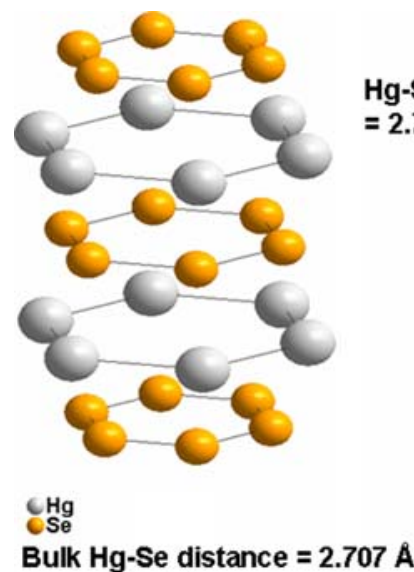

(a)

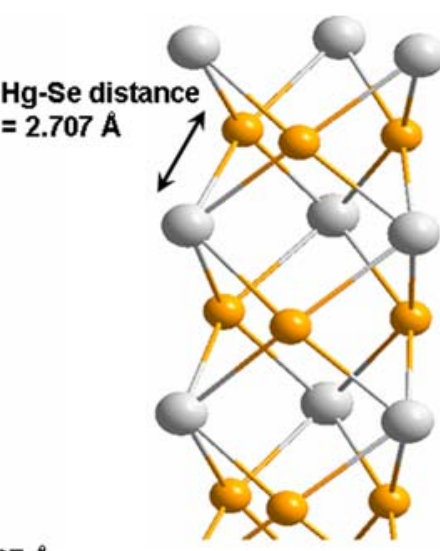

(b)
Fig. 3-Proposed structural model for the nanocluster in LTL channels. (a) The resultant atomic positions from the refinement; each refined $\mathrm{Se}$ and $\mathrm{Hg}$ site has 6 and 12 equal positions, respectively, in the channel. However, the refined occupancy, which agreed well with the reaction stoichiometry, TGA results, and the interatomic distances, does not permit the existence of atoms at all equal positions. (b) Chemically, the most favorable average structural model for the $\mathrm{HgSe}$ nanocluster for this atomic arrangement.

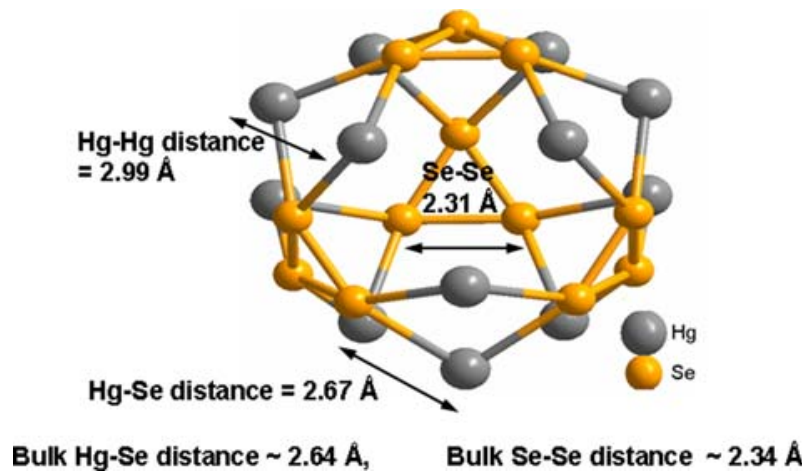

Fig. 4-Proposed structural model for the HgSe nanocluster in the pores of Nd-Y. The unit cell of Nd-Y contains eight large cages. According to the reaction stoichiometry (which agrees well with the refined occupancies ${ }^{[18]}$ ), only one-third of these sites can be filled. If we assume full occupancy in a cage, only 2.5 cages are filled out of eight total in the unit cell. Here, we have presented the most chemically satisfactory average structural model, which agrees with the preceding conditions, for the nanocluster. distance, $2.64 \AA$, and bulk Se-Se distance, $2.34 \AA$, respectively. These $\mathrm{HgSe}$ molecular structures are bonded to the framework through extra-framework oxygen atoms. In addition to the average $\mathrm{HgSe}$ clusters, we suggest that a significant disorder within the cluster contributes to the diffuse scattering.

\section{ATOMIC PDF RESULTS}

In Section III, diffuse scattering was subtracted from the XRDs, and the Bragg intensities were treated separately for the Rietveld refinement. As a result, the refinement yielded average structural models for the nanoclusters. The PDF studies were performed at ID-31 at the ESRF to understand the diffuse scattering present in our XRDs. A total PDF of the $\mathrm{NdY} / \mathrm{HgSe}$ system is given in comparison with the PDF of the extracted diffuse scattering in Figure 5. The PDF of the (Bragg + diffuse scattering) and the PDF of solely the diffuse scattering both give peaks at $\mathrm{Hg}-\mathrm{Se}$ and $\mathrm{Hg}-\mathrm{Hg}$ distances found in the Rietveld analysis, indicating displacive or substitutional disorder associated with the $\mathrm{Hg}$ and Se sites of the average structural model. Similar features were observed with the PDFs of the LTL/HgSe system. A displacive or substitutional disorder associated with the $\mathrm{Hg} / \mathrm{Se}$ sites was observed in these PDFs.

The hexagonal symmetry of the LTL/HgSe system allows each refined $\mathrm{Se}$ and $\mathrm{Hg}$ site to have 6 or 12 equivalent positions in the 1-D channel. However, the refined occupancy, which agrees well with the reaction stoichiometry and interatomic distances, does not permit $\mathrm{Hg}$ and $\mathrm{Se}$ atoms at all of the equivalent positions. Therefore, in the chemically most favorable refined structural model of the $\mathrm{HgSe}$ nanocluster, vacant atom sites exist in the filled channels. These vacancies can effectively contribute to the displacive or substitutional disorder observed in the PDF. If all of the channels in this framework are not filled with the guest molecules,

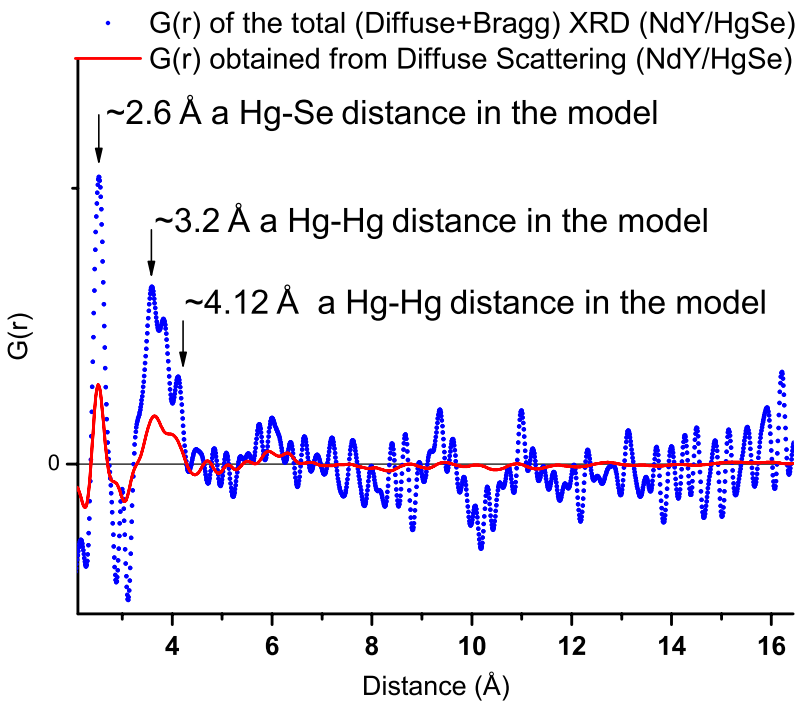

Fig. 5-Total PDF of the $\mathrm{NdY} / \mathrm{HgSe}$ system (dotted) in comparison with the PDF of the extracted diffuse scattering (line). 
correlations between neighboring channels can also contribute to diffuse scattering. However, 1-D LTL channels are too far from each other (center to center $18.4 \AA$ A) to have any direct correlations between individual channels.

The unit cell of $\mathrm{Nd}-\mathrm{Y}$ contains eight supercages. ${ }^{[27]}$ According to the reaction stoichiometry, which agrees well with the refined occupancies, ${ }^{[18]}$ only one-third of these sites can be filled. If we assume full occupancy in a single cage, only 2.5 cages can be filled out of eight total in the unit cell. These cages are $\sim 10.7 \AA$ (center to center) from each other. In the chemically most favorable refined model, vacant atom sites exist in the filled Nd-Y cages, which can contribute to displacive or substitutional disorder observed in its PDF.

\section{CONCLUSIONS}

A synthesis method was used to prepare compositionally well-defined samples, which were shown to contain $\mathrm{HgSe}$ nanoparticles within the zeolite channels. We did not observe any HgSe bulk phase peaks in XRD patterns. However, large intensity changes for the zeolite Bragg peaks were observed upon the addition of $\mathrm{HgSe}$, suggesting that $\mathrm{Hg}$ and $\mathrm{Se}$ take well-defined positions inside the zeolite cages. The absence of bulk phase $\mathrm{HgSe}$ diffraction peaks also confirms the confinement of $\mathrm{HgSe}$ in their zeolite frameworks. Furthermore, the observed changes in optical properties are consistent with those expected from constraining the $\mathrm{HgSe}$ nanoparticle size by the different channel sizes in Nd-Y and LTL; the narrower cluster in LTL is more blue-shifted than the average cluster in $\mathrm{NdY}$. Both $\mathrm{Nd}-\mathrm{Y} / \mathrm{HgSe}$ and LTL/ HgSe samples showed well-pronounced characteristics in the Raman spectra, which are clearly absent in the Raman spectra of empty zeolites and differ from those of bulk HgSe. Characteristic Raman features from Se-Se bonds in the LTL/HgSe system are assumed to be due to the presence of disorder within both pure selenium and HgSe clusters, as shown in Figure 3. The Se-Se features were identified with the previous knowledge of AlPO4-5/ Se single-crystal system, ${ }^{[13,10]}$ confirming that there is a reasonable fraction of $\mathrm{Se}-\mathrm{Se}$ bonds within the nanoclusters.

As a result of the Rietveld refinement, we have presented two distinct average structural models for the incorporated nanoclusters in the pores of zeolites, LTL and Nd-Y. To the best of our knowledge, this is the first detailed structural study done on $\mathrm{Nd}-\mathrm{Y} / \mathrm{HgSe}$ and LTL/ $\mathrm{HgSe}$ systems. The proposed models for the $\mathrm{HgSe}$ clusters both differ from the bulk $\mathrm{HgSe}$ structure (different symmetry than the bulk and 2 to $10 \mathrm{pct}$ change in bond lengths). It is our strong belief that the individual clusters possess substantial displacements or replacive (substitutional) disorder from the two average structures, thereby giving rise to the diffuse scattering. We strongly support this idea with our PDF studies, as described in the Section IV of this article.

Currently, we are working on a model for these nanoclusters that can incorporate both average molecular structure and the PDF more quantitatively. In addition to that, for a better understanding of the Raman spectra, we intend to grow HgSe nanostructures in the 1-D channels of AlPO4-5 single crystals. Qualitative X-ray structural studies of Se in AlPO4-5 single crystals (diffuse scattering associated with Se 1-D helical chain structures) have already been published by $\mathrm{Li}$ et al. ${ }^{[10]}$ We intend to extend these measurements to $\mathrm{Hg}$ Se and use diffractometer scans rather than photographic films.

\section{ACKNOWLEDGMENTS}

We thank the DOE/BES (Contract No. DE-FG0204ER46160) for their extended financial support of this work and the Robert A. Welch Foundation. We thank T. Vogt and Y. Lee (BNL/NSLS) for the use of the $\mathrm{X} 7 \mathrm{~A}$ beam line. We also gratefully acknowledge the ESRF for the use of PDF beam line ID-31. Jim Meen, Texas Center for Superconductivity at the University of Houston (TcSUH), performed SEM measurements, for which we thank him. This work is partly supported by the State of Texas through the Texas Center for Superconductivity at the University of Houston.

\section{REFERENCES}

1. A.L. Rogach, S.V. Kershaw, M. Burt, M. Harrison, A. Kornowski, A. Eychmüller, and H. Weller: Adv. Mater., 1999, vol. 11, pp. 55254.

2. X. Duan, Y. Huang, Y. Cui, J. Wang, and C.M. Lieber: Nature, 2001, vol. 409, pp. 66-69.

3. N. Hemon, Y. Wang, M.M. Eddy, G.D. Stucky, D.E. Cox, K. Moller, and T. Beid: Am. Chem. Soc., vol. 111 (2), pp. 530-40.

4. A. Goldbach, M. Grimsditch, L. Iton, and M.-L. Saboungi: J. Phys. Chem. B, 1997, vol. 101, pp. 330-34.

5. A. Goldbach and M.L. Saboungi: Eur. Phys. J.E., 2003, vol. 12, pp. $185-90$.

6. P. Armand, M.-L. Saboungi, D.L. Price, L. Iton, C. Cramer, and M. Grimsditch: Phys. Rev. Lett., 1997, vol. 79, pp. 2061-64.

7. A.L. Ankudinov, J.J. Rehr, J.J. Low, and S.R. Bare: J. Chem. Phys., 2002, vol. 116, pp. 1911-19.

8. T. Bein: MRS Bull., 2005, vol. 30, pp. 713-20.

9. G. Telbiz, O. Shvets, V. Vozny, and M. Brodyn: Study Surf. Sci. Catal., 2002, vol. 135, pp. 325-33.

10. I.L. Li, P. Launois, and Z.K. Tang: Appl. Surf. Sci., 2004, vol. 226, pp. 36-40.

11. A. Goldbach, M.-L. Saboungi, L. Iton, and D.L. Price: Chem. Commun., 1999, pp. 997-98.

12. T. Egami and S.J.L. Billinge: Underneath the Bragg Peaks: Structural Analysis of Complex Materials, Elsevier, New York, NY, 2003

13. V.V. Poborchii, A.V. Kolobov, J. Caro, V.V. Zhuravlev, and K. Tanaka: Phys. Rev. Lett., 1999, vol. 82, pp. 1955-58.

14. S.J.L. Billinge, V. Petkov, and Th. Proffen: Structure on Different Length Scales from Powder Diffraction: the Real-Space Pair Distribution Function (PDF) Technique, Commission on Powder Diffraction of the International Union of Crystallography Newsletter number 24, 2000.

15. V. Petkov, S.J.L. Billinge, S.D. Shastri, and B. Himmel: J. NonCryst. Solids, 2001, vols. 293-295, pp. 726-30.

16. S.J.L. Billinge: Z. Kristallogr., 2004, vol. 219, pp. 117-21.

17. S.J.L. Billinge: Local Structure from Diffraction, Plenum Press, New York, 1998.

18. A.M.M. Abeykoon, M. Castro-Colin, E.V. Anokhina, M.N. Iliev, W. Donner, A.J. Jacobson, and S.C. Moss: Phys. Rev. B, 2008, vol. 77, pp. 075333-1-10. 
19. W.E. Buhro and V.L. Colvin: Nat. Matter, 2003, vol. 2, pp. 138 39.

20. I. Goldbach, D. Meisel, J. Jonson, L.A. Curtiss, and M.L. Saboungi: J. Am. Chem. Soc., 1999, vol. 121, p. 1461.

21. Database of zeolite structures, http://www.iza-structure.org/ databases/.

22. R.M. Barrer and H. Villiger: Z. Kristallogr., 1969, vol. 128, pp. $352-70$.

23. J.J. Hriljac, M.M. Eddy, A.K. Cheetham, J.A. Donohue, and G.J. Ray: J. Solid State Chem., 1993, vol. 106, pp. 66-72.

24. C. Baerlocher, W.M. Meier, and D.H. Olson: Atlas of Zeolite Framework Types, Elsevier, New York, NY, 2001.
25. M.M.J. Treacy and J.B. Higgins: Collection of Simulated XRD Powder Patterns for Zeolites, Elsevier, New York, NY, 2001.

26. D.E. Cox and A.P. Wilkinson: in Resonant Anomalous X-Ray Scattering: Theory and Applications, G. Materlik, C.J. Sparks, and K. Fischer, eds., Elsevier Science B.V., New York, 1994, pp. 195-219.

27. J.G. Nery, M.V. Giotto, Y.P. Mascarenhas, D. Cardoso F. Maria, Z.Z. Eduardo, and F. Sousa-Aguiar: Microporous Mesoporous Mater., 2000, vol. 41, pp. 281-93. 